\title{
Lacunary sequences that do not influence the uniqueness of solution of the inverse Borg- Levinson problem
}

\author{
Irina I. Kinzina \\ Nosov Magnitogorsk State Technical University, \\ Magnitogorsk city, Chelyabinsk region, Russian Federation \\ kinzina@mail.ru
}

\author{
Larisa V. Smirnova \\ Nosov Magnitogorsk State Technical University, \\ Magnitogorsk city, Chelyabinsk region, Russian Federation \\ smirnova20@bk.ru
}

\author{
Olga A. Torshina \\ Nosov Magnitogorsk State Technical University, \\ Magnitogorsk city, Chelyabinsk region, Russian Federation \\ olganica@mail.ru
}

\begin{abstract}
Here we present the inverse problem of spectral analysis with the Dirichlet boundary conditions for the Laplace operator with a potential defined in a bounded domain of multidimensional space. The uniqueness theorem of the recovery of potential in the inverse Borg-Levinson problem with boundary conditions of the first kind is proved. A possible mathematical model of the recovery of potential is built on the basis of this theorem using incomplete spectrum.
\end{abstract}

Keywords-inverse problem; inverse problem of spectral analysis; the Laplace operator; potential; recovery of potential; the Dirichlet problem; lacunary sequence

\section{INTRODUCTION}

The inverse problems of spectral analysis are problems of reconstructing a linear operator by means of its given spectral characteristics (spectrums under different boundary conditions, spectral function, scattering data, etc).

The existence theorems of potential in some inverse problems of spectral analysis are known, for example, for ordinary differential equations, for powers of the Laplace operator with a potential on a rectangle. Therefore it is interesting to solve the uniqueness problem of the recovery of potential by means of given spectral characteristics in a bounded domain $\Omega$ at least from $R^{2}$.

There is a formulation of the Borg-Levinson problem with the Dirichlet boundary conditions in H. Isozaki's paper [1]. In this paper, following $\mathrm{H}$. Isozaki we consider such formulation of the Borg-Levinson problem.

In [1] H. Isozaki concluded that the lack of knowledge about the finite number of spectral data does not influence the uniqueness of the recovery of potential. Similar spectral problems were considered in [2]-[7], [10]-[17]. In some papers the authors proves the uniqueness theorems of the recovery of potential in the Borg-Levinson problem by means of incomplete spectral data of various types.

\section{RESULTS AND DISCUSSION}

Let $\Omega$ be a bounded domain in $R^{N} \quad(N \geq 2)$ with boundary $S \in C^{\infty}$. We consider the Dirichlet boundary value problem with function $q \in C^{\infty}(\bar{\Omega})$ and spectral parameter $\lambda$

$$
\left\{\begin{array}{l}
-(\Delta u)(x)+q(x) u(x)=\lambda u(x), x \in \Omega, \\
\left.u(x)\right|_{S}=0 .
\end{array}\right.
$$

The solution of this problem is a function from $C^{\infty}(\bar{\Omega})$ that satisfies (1) everywhere on $\bar{\Omega}$.

Let eigenvalues $\mu_{1}, \mu_{2}, \mathrm{~K}$ of problem (1) be numbered in increasing order, and let $m_{t}=m_{t}(q)$ be multiplicity of eigenvalue $\mu_{t}(q)$. By $u_{j t}, 1 \leq j \leq m_{t}$, denote orthonormal eigenfunctions that correspond to eigenvalues $\mu_{t}$. These sets of functions are determined ambiguously, so we consider their specific classes $W_{t}$.

We introduce the Neumann operator $N: L_{2}(\Omega) \rightarrow L_{2}(S)$ by the equality

$$
N(\lambda, q) V=\left.\frac{\partial V}{\partial v}\right|_{S} .
$$

Consider problem (1) with potentials $q_{1}, q_{2} \in C^{\infty}(\bar{\Omega})$. Define scattering functions by the rule

$$
F\left(\lambda, \omega, \theta ; q_{j}\right)=\int_{S}\left(N\left(\lambda, q_{j}\right) \varphi_{\lambda, \omega}\right)(x) \overline{\varphi_{\lambda,-\theta}(x)} d S_{x},
$$

where $S_{x}$ is a surface area element, $\varphi_{\lambda, \omega}(x)=\exp (i \sqrt{\lambda} \omega \cdot x)$, $\lambda \in C /(-\infty ; 0), \quad \omega, \theta \in S^{N-1}, \quad i \quad$ is an imaginary unit, $\omega \cdot x=\sum_{r=1}^{N} \omega_{k} x_{k}, j=1,2$.

We know that

$$
\begin{gathered}
F\left(\lambda, \omega, \theta ; q_{j}\right)= \\
=-\frac{\lambda}{2}(\theta-\omega)^{2} \int_{\Omega} \exp (-i \sqrt{\lambda}(\theta-\omega) x) d x+\int_{\Omega} \exp (-i \sqrt{\lambda}(\theta-\omega) x) q_{j}(x) d x- \\
=\int_{S}\left(-\Delta+q_{j}-\lambda\right)^{-1}\left(q_{j} \varphi_{\lambda, \omega}\right)(x) \cdot \overline{\varphi_{\lambda,-\theta}(x)} d S_{x} .
\end{gathered}
$$


Let $\xi \in R^{N}, \xi \neq 0$ be an arbitrary fixed vector. Choose $\eta \in R^{N},|\eta|=1$, so that $\eta$ and $\xi$ are orthogonal. Let $n$ be a sufficiently large natural number. Define such sequences:

$$
\begin{aligned}
& c_{n}=\left(1-\frac{|\xi|^{2}}{4 n^{2}}\right)^{\frac{1}{2}}, \quad \theta_{n}=c_{n} \eta+\frac{\xi}{2 n}, \\
& \omega_{n}=c_{n} \eta-\frac{\xi}{2 n}, \quad l_{n}=(n+i)^{2} .
\end{aligned}
$$

Taking into account such choice of variables $l_{n}, \theta_{n}, \omega_{n}$, $c_{n}$, we have

$$
\lim _{n \rightarrow \infty} F\left(l_{n}, \theta_{n}, \omega_{n} ; q_{j}\right)=-\frac{|\xi|^{2}}{2} \int_{\Omega} \exp (-i x \cdot \xi) d x+\int_{\Omega} \exp (-i x \cdot \xi) q_{j}(x) d x .
$$

A certain power of Neumann operator is an integral operator. Kernel of Neumann operator is formally given by the following equality:

$$
\operatorname{ker} N\left(\lambda, q_{j}\right)(x, y)=\sum_{t=1}^{\infty} \frac{\partial u_{t}\left(q_{j}\right)}{\partial v}(x) \overline{\frac{\partial u_{t}\left(q_{j}\right)}{\partial v}(y)} \cdot\left(\mu_{t}\left(q_{j}\right)-\lambda\right)^{-1},
$$

where $x, y \in S$.

Showing the validity of condition

$$
\left\|\operatorname{ker} N\left(\lambda, q_{1}\right)-\operatorname{ker} N\left(\lambda, q_{2}\right)\right\| \rightarrow 0 \text { as } n \rightarrow \infty,
$$

and using equality (3), we get

$$
\lim _{n \rightarrow \infty}\left|F\left(l_{n}, \theta_{n}, \omega_{n} ; q_{1}\right)-F\left(l_{n}, \theta_{n}, \omega_{n} ; q_{2}\right)\right|=0,
$$

and therefore $q_{1}(x)=q_{2}(x)$ for any $x \in \bar{\Omega}$.

Requirement (4) is satisfied if the following condition holds:

$$
\lim _{n \rightarrow \infty}\left\|\sum_{t=T+1}^{\infty} \frac{\frac{\partial u_{t}\left(q_{1}\right)}{\partial v}(x) \frac{\overline{\partial u_{t}\left(q_{1}\right)}}{\partial v}(y)\left(\mu_{t}\left(q_{2}\right)-\mu_{t}\left(q_{1}\right)\right)}{\left(\mu_{t}\left(q_{1}\right)-l_{n}\right)\left(\mu_{t}\left(q_{2}\right)-l_{n}\right)}\right\|_{L_{2}(s \times s)}=0 .
$$

Academician V.A. Ilyin and I.A. Shishmarjov showed in [8] that

$$
\left\|\frac{\partial u_{t}}{\partial v}\left(q_{j}\right)\right\|_{L_{2}(S)}=O\left(\left[\mu_{t}\left(q_{j}\right)\right]^{\frac{N}{4}}\right), j=1,2 .
$$

Using Hörmander's theorem [9], we have

$$
\mu_{t} \sim C_{3} \cdot t^{\frac{2}{N}} \text { as } t \rightarrow \infty .
$$

Therefore, we state

$$
\left\|\frac{\partial u_{t}}{\partial v}\left(q_{j}\right)\right\|_{L_{2}(S)}=O\left(t^{\frac{1}{2}}\right), j=1,2 .
$$

Thus, requirement (5) is valid if

$$
\lim _{n \rightarrow \infty} \sum_{k=1}^{\infty} \frac{t_{k}}{\left|\mu_{t_{k}}\left(q_{1}\right)-l_{n}\right|\left|\mu_{t_{k}}\left(q_{2}\right)-l_{n}\right|}=0 .
$$

Suppose the subsequences of eigenvalues of problems (1) satisfy condition

$$
\forall k \in N\left(\frac{t_{k+1}}{t_{k}} \geq \lambda>1\right) .
$$

Such sequences are called lacunary.

In addition, consider arbitrary strictly increasing sequences of natural numbers which are satisfying the condition

$$
\lim _{k \rightarrow \infty} \frac{t_{k+1}-t_{k}}{\sqrt{t_{k}}}=+\infty .
$$

We can show that requirement (5) is true with such choice of sequence.

The replacement of natural number $n$ by a positive real number in (2) does not disturb equality (3).

Let $l_{n_{k}}$ be such that

$$
\operatorname{Re} l_{n_{k}}=\frac{\mu_{t_{k+1}}\left(q_{1}\right)-\mu_{t_{k}}\left(q_{1}\right)}{2} .
$$

Let us consider (6).

Fix $n_{s}$. Then $\mu_{t_{s}}<l_{n_{s}}$, and $\mu_{t_{s+1}}>l_{n_{s}}$.

$$
\begin{gathered}
\sum_{k=1}^{\infty} \frac{t_{s+k}}{\left|\mu_{t_{s+k}}\left(q_{1}\right)-l_{n}\right|\left|\mu_{t_{s+k}}\left(q_{2}\right)-l_{n}\right|} \leq \\
\leq|O(1)| \sum_{k=1}^{\infty} \frac{t_{s+k}}{t_{s+k}^{2}}=|O(1)| \sum_{k=1}^{\infty} \frac{1}{t_{s+k}} \leq|O(1)| \sum_{k=1}^{\infty} \frac{1}{\lambda^{k} t_{s}} \\
=|O(1)| \frac{1}{t_{s}} \sum_{k=1}^{\infty} \frac{1}{\lambda^{k}}=\left|O\left(\frac{1}{t_{s}}\right)\right| \rightarrow 0
\end{gathered}
$$

as $s \rightarrow \infty$.

Using the properties of lacunary sequences, we obtain

$$
\begin{gathered}
\sum_{p=1}^{s} \frac{t_{p}}{\left|\mu_{t_{p}}\left(q_{1}\right)-l_{n}\right|\left|\mu_{t_{p}}\left(q_{2}\right)-l_{n}\right|} \leq|O(1)| \sum_{k=1}^{\infty} \frac{t_{p}}{\left.\frac{t_{s+1}+t_{s}}{2}-t_{p}\right)^{2}} \leq \\
\leq \frac{t_{s}}{\left(\frac{t_{s}(\lambda-1)}{2}\right)^{2}}+\frac{t_{s-1}}{(\lambda-1)^{2}\left(\frac{t_{s}}{2}+t_{s-1}\right)^{2}}+\Lambda+ \\
+\frac{t_{1}}{(\lambda-1)^{2}\left(\frac{t_{s}}{2}+t_{s-1}+\Lambda+t_{1}\right)^{2}} \leq \\
\leq \frac{4 t_{s}}{(\lambda-1)^{2} t_{s}^{2}}+\frac{4 t_{s-1}}{(\lambda-1)^{2}(\lambda+1)^{2} t_{s-1}^{2}}+\Lambda+
\end{gathered}
$$




$$
\begin{gathered}
+\frac{4 t_{1}}{(\lambda-1)^{2}\left(\lambda^{s-1}+\lambda^{s-2}+\Lambda+\lambda+1\right)^{2} t_{1}^{2}}= \\
=\frac{4 t_{s}}{(\lambda-1)^{2} t_{s}^{2}}+\frac{4 t_{s-1}}{\left(\lambda^{2}-1\right)^{2} t_{s-1}^{2}}+\Lambda+\frac{4 t_{1}}{\left(\lambda^{s}-1\right)^{2} t_{1}^{2}}= \\
=4 \sum_{p=1}^{s} \frac{1}{\left(\lambda^{s-p+1}-1\right)^{2} t_{p}} \leq \\
\leq \frac{4}{(\lambda-1)^{2}} \sum_{p=1}^{s} \frac{1}{t_{p}}=\frac{4}{(\lambda-1)^{2}} O\left(\frac{1}{t_{s}}\right) \rightarrow 0
\end{gathered}
$$

as $s \rightarrow \infty$.

There is a similar proof for three-dimensional space. For this case we shall use the asymptotics

$$
\mu_{t} \sim C_{3} \cdot t^{\frac{2}{3}}, C_{3}>0 .
$$

If we have $N$-dimensional space $(N \geq 2)$, then we will require "the similarity" of problems (1) with potentials $q_{1}$ and $q_{2}$. It ensures the uniqueness of the recovery of potential.

Let $l_{n_{k}}$ be such that

$$
\operatorname{Re} l_{n_{k}}=\frac{\mu_{t_{k+1}}\left(q_{1}\right)-\mu_{t_{k}}\left(q_{1}\right)}{2} .
$$

Let us consider (6).

Fix $n_{s}$. Then $\mu_{t_{s}}<l_{n_{s}}$, and $\mu_{t_{s+1}}>l_{n_{s}}$.

$$
\begin{gathered}
\sum_{k=1}^{\infty} \frac{t_{s+k}^{2 \alpha}}{\left|\mu_{t_{s+k}}\left(q_{1}\right)-l_{n}\right|\left|\mu_{t_{s+k}}\left(q_{2}\right)-l_{n}\right|} \leq|O(1)| \sum_{k=1}^{\infty} \frac{t_{s+k}^{2 \alpha}}{t_{s+k}^{\prime N}}= \\
=|O(1)| \sum_{k=1}^{\infty} \frac{1}{t_{s+k}^{\frac{4}{N}-2 \alpha}} \leq|O(1)| \sum_{k=1}^{\infty} \frac{1}{\lambda^{k\left(\frac{4}{N}-2 \alpha\right)} \frac{4}{t_{N}}-2 \alpha}= \\
=|O(1)| \frac{1}{t_{s}^{\frac{4}{N}-2 \alpha}} \sum_{k=1}^{\infty} \frac{1}{\lambda^{k\left(\frac{4}{N}-2 \alpha\right)}}=\left|O\left(\frac{1}{t^{\frac{4}{N}-2 \alpha}}\right)\right| \rightarrow 0
\end{gathered}
$$

as $s \rightarrow \infty$, since $\frac{4}{N}-2 \alpha>0, \lambda>1$.

Using the inequality for positive $a$ and $b$

$$
a+b \geq C_{\gamma} \cdot a^{\gamma} \cdot b^{1-\gamma}, C_{\gamma}=\text { const }>0,0<\gamma<1,
$$

we have

$$
\begin{gathered}
\sum_{p=1}^{s} \frac{t_{p}^{2 \alpha}}{\left|\mu_{t_{p}}\left(q_{1}\right)-l_{n}\right| \mu_{t_{p}}\left(q_{2}\right)-l_{n} \mid} \leq \\
\leq \frac{|O(1)|}{\left(t_{s+1}^{2 / N}+t_{s}^{2 / N}\right)^{1-\gamma}} \sum_{k=1}^{s} \frac{t_{p}}{\left(\frac{t_{s+1}^{2 / N}+t_{s}^{2 / N}}{2}-t_{p}\right)^{2 \gamma}} \leq
\end{gathered}
$$

$$
\begin{gathered}
\leq \frac{|O(1)|}{\left(t_{s+1}^{2 / N}+t_{s}^{2 / N}\right)^{1-\gamma}} \sum_{k=1}^{s} \frac{1}{t_{p}^{\frac{4 \gamma}{N}-2 \alpha}(s+1-k)^{2}\left(\lambda^{\frac{2}{N}}-1\right)^{2}} \leq \\
\leq \frac{O(1)}{\left(t_{s+1}^{\frac{2}{N}}+t_{s}^{\frac{2}{N}}\right)^{1-\gamma}\left(\lambda^{\frac{2}{N}}-1\right)^{2}} \cdot \sum_{p=1}^{s} \frac{1}{(s+1-p)^{2}} \leq \\
\leq \frac{\left.O(1)^{\left(\frac{2}{N}\right.}+t_{s}^{\frac{2}{N}}\right)^{1-\gamma}\left(\lambda^{\frac{2}{N}}-1\right)^{2}}{s-1} \leq \frac{s-1}{s} \rightarrow 0 \\
s^{\frac{2(1-\gamma)}{N}+1}
\end{gathered}
$$

as $s \rightarrow \infty$.

Thus, (6) and consequently (4) hold.

\section{CONCLUSION}

Theorem 1. Let $\Omega$ be a bounded domain in $R^{N}, N=2,3$, with boundary $S \in C^{\infty}$, apart from possibly points $\left\{\mu_{t_{k}}\left(q_{j}\right)\right\}_{k=1}^{\infty}, j=1,2$. These points are subsequences of the eigenvalues of problems (1) with potentials $q_{j}$. Let they have property (7) or (8). If the following conditions for the orthonormal eigenfunctions of the Dirichlet problems (1) with potentials $q_{1}$ and $q_{2}$ hold:

a) $W_{t}\left(q_{1}\right)=W_{t}\left(q_{2}\right)$ as $t \neq t_{k}$;

b) $\mu_{t}\left(q_{1}\right)=\mu_{t}\left(q_{2}\right)$ as $t \neq t_{k}$,

then for any $x \in \bar{\Omega}$ the equality $q_{1}(x)=q_{2}(x)$ holds.

Using theorem 1, we propose a model of the recovery of potential (MRP 1).

MRP 1. Let $\Omega$ be a bounded domain in $R^{N}, N=2,3$, with boundary $S \in C^{\infty}$. Suppose we know all eigenvalues, apart from possibly a finite set, and values of the eigenfunctions on boundary $S$ in problem (1) with potential $q_{1}$. If the following conditions for problem (1) with potentials $q_{2}$ hold:

a) there exists a natural number $T$ such that for every number $t \geq T$ we have $W_{t}\left(q_{1}\right)=W_{t}\left(q_{2}\right)$;

б) $\mu_{t}\left(q_{1}\right)=\mu_{t}\left(q_{2}\right)$ as $t \neq t_{k}$, where subsequences $\left\{\mu_{t_{k}}\left(q_{2}\right)\right\}_{k=1}^{\infty}$ of the eigenvalues of problems (1) with potentials $q_{2}$ have property (7) or (8),

then for any $x \in \bar{\Omega}$ the equality $q_{1}(x)=q_{2}(x)$ holds.

Thus, the potential $q_{2}$ in problem (1) is recovered.

Theorem 2. Let $\Omega$ be a bounded domain in $R^{N}, N \geq 2$, with boundary $S \in C^{\infty}$. Let subsequences $\left\{\mu_{t_{k}}\left(q_{j}\right)\right\}_{k=1}^{\infty}$, $j=1,2$, of the eigenvalues of problems (1) with potentials 
$q_{j}$ have property (7). If the following conditions for the orthonormal eigenfunctions of the Dirichlet problems (1) with potentials $q_{1}$ and $q_{2}$ hold:

a) there exists a natural number $T$ such that for every number $t \geq T$ we have $W_{t}\left(q_{1}\right)=W_{t}\left(q_{2}\right)$;

b) $\mu_{t}\left(q_{1}\right)=\mu_{t}\left(q_{2}\right)$ as $t \neq t_{k}$;

c) $\left|\mu_{t_{k}}\left(q_{1}\right)-\mu_{t_{k}}\left(q_{2}\right)\right|=O\left(t_{k}^{2 \alpha-1}\right)$, where $\alpha<\frac{2}{N}$,

then for any $x \in \bar{\Omega}$ the equality $q_{1}(x)=q_{2}(x)$ holds.

Theorems 1 and 2 can be considered as the uniqueness theorems of the recovery of potential in the inverse Dirichlet problem.

Using theorem 2 we construct a model of the recovery of potential (MRP 2) similar to MRP 1.

\section{REFERENCES}

[1] Isozaki H. Some remarks on the multidimensional Borg-Levinson theorem. J. Math. Kyoto Univ. (JMK YAZ), 1991, vol. 31, no. 3, pp. 743-753.

[2] Dubrovskii V.V., Smirnova L.V. On the issue about the recovery of potential in the inverse Robin problem. Izvestija vuzov. Matematika [Proceedings of high schools. Mathematics], 2002, no. 7 (482), pp. 8 13. (in Russian)

[3] Smirnova L.V. On the issue about a mathematical model of the recovery of smooth potentials in the inverse Dirichlet problem for $n$ dimensional case. Matematicheskoe i programmnoe obespechenie sistem v promyshlennoj i social'noj sferah [Software of systems in the industrial and social fields], 2013, no. 1 (3), pp. 11-17. (in Russian)

[4] Sadovnichii V.A., Dubrovskii V.V., Smirnova L.V. Uniqueness of solutions to inverse eigenvalue problems. Doklady Mathematics, 2000 , vol. 61, no. 1, pp. 67-69.

[5] Dubrovskii V.V., Smirnova L.V. On the uniqueness of solution of the inverse spectral problems for equations of mathematical physics. Fundamental'naja i prikladnaja matematika [Fundamental and Applied Mathematics], 1999, vol. 5, no. 2, pp. 411-416. (in Russian)

[6] Smirnova L.V. Question of uniqueness of recovery of potential by spectrum in the inverse Borg Levinson problem with Robin boundary conditions. Journal of Computational and Engineering Mathematics, 2015, vol. 2, no. 4, pp. 73-83.

[7] Smirnova L.V., Torshina O.A. Infinite sequences not affecting the unique recovery of the potential. Applied and Fundamental Studies. Proceedings of the 4th International Academic Conference. November 29-30, 2013, St. Louis, USA. Publishing House Science and Innovation Center, Ltd., 2013.

[8] Ilyin V.A., Shishmarjov I.A. Uniform estimates in a closed domain for eigenfunctions of elliptic operator and their derivatives. Izvestija AN SSSR. Serija: Matematika [Proceedings of the USSR Academy of Sciences. Series: Mathematics], 1960, vol. 24, pp. 883-896. (in Russian)

[9] Hörmander L. The spectral function of elliptic operator. Acta math., 1968, vol. 121, pp. 193-218.

[10] Kinzina I.I. Calculation of eigenvalues of perturbed discrete operators. Cheljabinskij fiziko-matematicheskij zhurnal [Chelyabinsk Physics and Mathematics Journal], 2008, no. 6, pp. 34-43. (in Russian)

[11] Kinzina I.I. Computing eigenvalues of discrete self-adjoint operator perturbed by bounded operator. Izvestija vuzov. Matematika
[Proceedings of high schools. Mathematics], 2008, no. 6, pp. 16-24. (in Russian)

[12] Kinzina I.I. Algoritm nahozhdenija sobstvennyh chisel vozmushhennyh diskretnyh samosoprjazhennyh operatorov. Diss. [The algorithm for the finding eigenvalues of perturbed discrete selfadjoint operators. Diss.]. Magnitogorsk, 2006. 168 p. (in Russian)

[13] Kinzina I.I. Calculating corrections of perturbation theory of discrete operators. Vestnik Magnitogorskogo gosudarstvennogo universiteta [Bulletin of Magnitogorsk State University], 2006, no. 9, pp. 53-63. (in Russian)

[14] Smirnova L.V., Kushkumbaeva A.S., Torshina O.A. To the question of the uniqueness of the reduction potential in the inverse problem of the Borg-Levinson. Proceedings of the 2016 conference on information technologies in science, Management, Social sphere and medicine (itsmssm). Book series: Acsr-Advances in Comptuer Sclence Research, 2016. Vol. 51 - pp. 494-497.

[15] Torshina O.A. Formula for first regularized trace of the LaplaceBochner operator with potential on projective plane. Voronezhskaja zimnjaja matematicheskaja shkola [Voronezh Winter Mathematical School], 2004, pp. 104-105. (in Russian)

[16] Torshina O.A. About the trace of differential operator with potential on projective plane. Vestnik Cheljabinskogo gosudarstvennogo universiteta [Bulletin of Chelyabinsk State University], 2003, vol. 3, no. 3, pp. 178-191. (in Russian)

[17] Torshina O.A. Formula for first regularized trace of the LaplaceBeltrami operator with nonsmooth potential on projective plane. Vestnik Samarskogo gosudarstvennogo tehnicheskogo universiteta. Serija: Fiziko-matematicheskie nauki [Bulletin of Samara State Technical University. Series: Physics and Mathematics], 2006, no. 4, pp. 32-40. (in Russian) 